\title{
The use of Fourier transform mid infrared (FT-MIR) spectroscopy for detection and quantification of adulteration in virgin coconut oil.
}

\begin{abstract}
Currently, the authentication of virgin coconut oil (VCO) has become very important due to the possible adulteration of VCO with cheaper plant oils such as corn (CO) and sunflower (SFO) oils. Methods involving Fourier transform mid infrared (FT-MIR) spectroscopy combined with chemometrics techniques (partial least square (PLS) and discriminant analysis (DA)) were developed for quantification and classification of CO and SFO in VCO. MIR spectra of oil samples were recorded at frequency regions of $4000-650 \mathrm{~cm}-1$ on horizontal attenuated total reflectance (HATR) attachment of FTIR. DA can successfully classify VCO and that adulterated with $\mathrm{CO}$ and SFO using 10 principal components. Furthermore, PLS model correlates the actual and FTIR estimated values of oil adulterants (CO and SFO) with coefficient of determination (R2) of 0.999.
\end{abstract}

Keyword: FT-MR spectroscopy; Virgin coconut oil; Corn oil; Sunflower oil; Adulteration. 